\title{
Control of electronic populations in porphycene with chirped ultrashort pulses
}

\author{
Piotr Ciąćka, Piotr Fita, and Czesław Radzewicz \\ ${ }^{1}$ Institute of Experimental Physics, Faculty of Physics, University of Warsaw, Pasteura 5, 02-093 Warszawa
}

Received September 03, 2015; accepted September 29, 2015; published September 30, 2015

\begin{abstract}
The degree of optical excitation of porphycene in solution can be controlled by manipulating a linear chirp of high intensity ultrashort laser pulses while keeping their energies and spectra constant Transient absorption, which reflects molecular populations in the ground and the first singlet excited state, varies by over $100 \%$ between optimal positively and negatively chirped excitation pulses. The results are discussed invoking coherent vibrational wavepacket dynamics.
\end{abstract}

Porphycene, a synthetic organic molecule (Fig. 1), attracts considerable interest of researchers primarily due to intramolecular double hydrogen transfer occurring near its internally-positioned nitrogens [1]. This process has been successfully studied with pump-probe ultrafast techniques, yielding information on both ground- and excited-state hydrogen transfer rates in a variety of porphycene derivatives $[2,3]$.

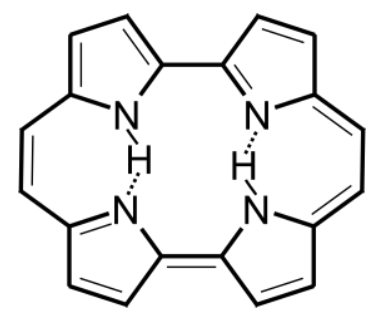

Fig. 1. Structure of a porphycene molecule.

In this work, we focus on population control in porphycene and show that the transient absorption signal related to populations in both ground and excited state can be modified by more than $100 \%$ by varying only the linear chirp of a high intensity pump pulse while keeping both its energy and spectrum constant. This result comprises an example of coherent control, in which coherent properties of electromagnetic fields are mapped onto matter wave functions. Exploiting either interference effects of continuous wave radiation or time-ordered interactions with short pulses, the evolution of a system can be steered towards the desired product [4]. Our approach employs interactions of pulses with coherent vibrational wavepackets, mirroring the strategy used previously to alter the fluorescence yield [5] or vibrational coherence [6] in a laser dye LD690. In effect,

*E-mail: pciacka@fuw.edu.pl interactions taking place during the first few hundreds of femtoseconds after excitation can be used to affect the behaviour of a molecule nanoseconds later.

A prerequisite for the aforementioned phenomena to occur is a strong pumping field to fulfil the saturated absorption condition, when more than $50 \%$ of illuminated molecules are excited. It enables multiple interactions of photons with each molecule. To facilitate that, our femtosecond transient absorption setup used in hydrogen transfer studies [3] was accordingly modified. Briefly, two non-collinear optical parametric amplifiers at the output of a femtosecond Ti:Sapphire regenerative amplifier served as independently-tunable sources of beams for pumping and probing the sample. A commercial acousto-optic programmable dispersive filter (AOPDF) [7] acted as a spectral phase shaper that allowed pump pulses to be both positively and negatively chirped. Out of necessity to compensate the positive dispersion of AOPDF and other optical elements, the obtainable negative chirp before the onset of undesirable changes to the pulse spectrum was around $-4000 \mathrm{fs}^{2}$, while the positive dispersion range was in excess of $7000 \mathrm{fs}^{2}$. AOPDF synchronized with the regenerative amplifier also served as a chopper blocking every other pump pulse, a feature used to reduce noise related to pulse energy fluctuations.

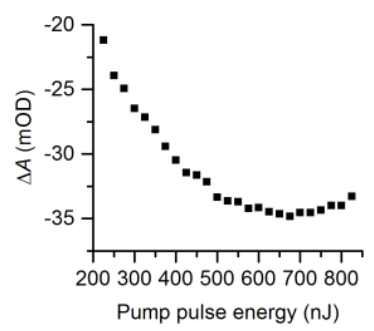

Fig. 2. Dependence of the transient absorption signal on the pump pulse energy.

Both pump and probe beams were focused with a single $100 \mathrm{~mm}$ lens. While no direct beam waist diameter measurements were carried out, on the basis of Gaussian beam propagation we estimate that even for $515 \mathrm{~nJ}$, that is the lower of two pulse energies used in the experiments, the excitation of molecules in the sample solution approached $75 \%$, well in the saturated region. The plot in 
Fig. 2 showing the dependence of the transient absorption signal on pump pulse energy is clearly nonlinear, supporting the saturated absorption picture.

All measurements were performed on porphycene solution in a mixture of toluene and paraffin oil in a $1 \mathrm{~mm}$ cuvette with both pump and probe wavelengths set to $633 \mathrm{~nm}$, which corresponds to the excitation to the lowest singlet excited state.

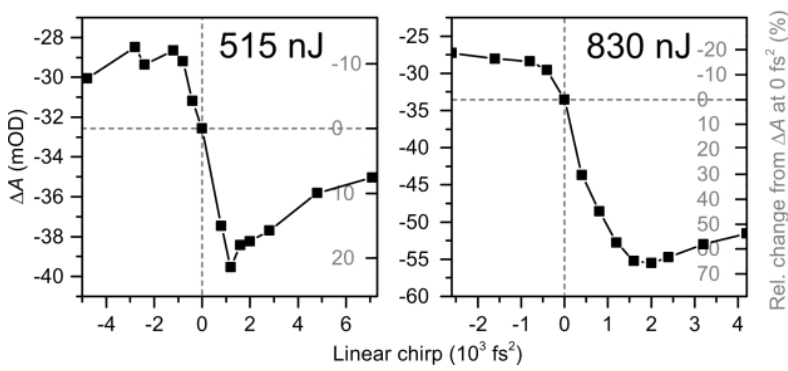

Fig. 3. Transient absorption as a function of linear chirp for two pump pulse energies, both corresponding to saturated absorption regime.

Figure 3 shows changes in the transient absorption signal after applying different linear chirps to pump pulses of energy $515 \mathrm{~nJ}$ and $830 \mathrm{~nJ}$. With respect to the shortest pulse at $0 \mathrm{fs}^{2}$, close to and for convenience referred to as transform-limited, a negatively chirped pulse near $-1600 \mathrm{fs}^{2}$ decreases $\Delta A$ signal by up to $20 \%$, while the signal for a positively chirped pulse of $1200 \mathrm{fs}^{2}$ is almost $70 \%$ higher. Overall, transient absorption changes by slightly more than $100 \%$ when switching between optimally - positively and negatively - chirped pulses.

The transient absorption signal at pump and probe wavelengths tuned to the transition between the ground and the first singlet excited state at $633 \mathrm{~nm}$ is dominated by two features. Firstly, the ground state is depleted, causing increased transmission of the probe beam and appearance of negative $\Delta A$ signal. Secondly, stimulated emission from the excited state amplifies probe light, yielding even more negative signal. Therefore, transient absorption is related to the population of "holes" in the ground state and equal population of molecules in the excited state.
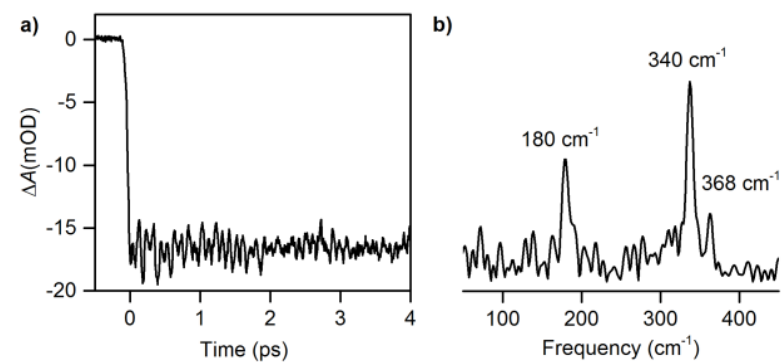

Fig. 4. a) Modulation of transient absorption kinetics due to excitation of coherent vibrational wavepackets, b) Power spectrum of the transient absorption kinetic trace.
It is well established [8] that a short, broadband pulse not only populates several vibrational sublevels of an excited-state electronic potential, but also imparts definite phase relationships among wave functions of those sublevels, launching a non-stationary coherent wavepacket. Its temporal evolution corresponds to molecular vibrations in the classical sense, that is - to a periodic displacement of the nuclei.

Nuclear motion impacts electronic transition frequencies, leading to characteristic oscillations in transient absorption kinetics when pumped and probed by pulses shorter than the period of a given vibrational mode. An example for porphycene recorded for non-saturated conditions at the same pump and probe wavelengths as used in population control experiments is shown in Fig. 4a. In the power spectrum of a trace (Fig. 4b) one finds porphycene's characteristic vibrational frequencies [9]. The oscillation period of the retrieved mode of the highest frequency is $91 \mathrm{fs}$.
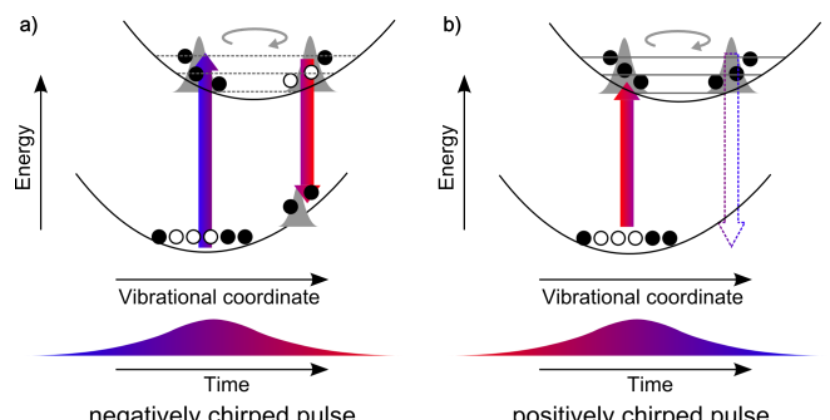

Fig. 5. Wavepacket (grey Gaussians) and population (black dots) dynamics during excitation by a) negatively chirped, b) positively chirped pulses under saturated absorption condition.

The existence of such a coupling between temporal properties of the pump pulse and evolution of a vibrational wavepacket comes into play especially in the nonlinear (i.e. saturated absorption) regime. For a high intensity pump pulse each molecule can interact with more than one photon. Therefore, for a negatively chirped pulse (Fig. 5a), in the first interaction the blue (leading) part of the pump pulse depletes the ground state as well as launches a coherent wavepacket in the excited state. A second interaction with the red (trailing) part of the pulse occurs later in time, when the wavepacket reaches a point where the stimulated emission cross section is large for red-shifted wavelengths. The molecule is transferred back to the ground state, decreasing the population of ground state "holes" and excited molecules, therefore leading to a smaller change of absorption. The process is accompanied by the formation of an additional wavepacket in the ground state which can be observed as a stronger modulation of transient absorption kinetics for negatively chirped pump pulses [10]. 
On the contrary, the second interaction with a positively chirped pulse (Fig. 5b) involves a blue-shifted photon. Since it is farther from the resonance condition at the time of interaction, stimulated emission will be inhibited, preserving the populations established after the first interaction. Effectively, the system interacts with a positively chirped pulse as if it was a one-photon interaction, even at intensities otherwise corresponding to saturated-absorption conditions.

A middle-of-the-road situation exists for transformlimited pulses. Some excited state population is dumped back to the ground state after two virtually simultaneous interactions before significant progression of a vibrational wavepacket takes place.

For pulses longer than optimal ones, the effect diminishes since the ability of long pulses to excite coherent wavepackets is decreased. Additionally, with the increase of a negative chirp, instantaneous frequencies will get out of synchronization with wavepacket-governed transient resonances.

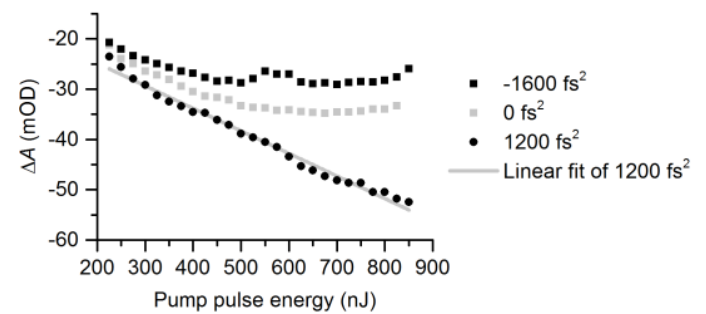

Fig. 6. Transient absorption dependence on pump pulse energy for transform-limited as well as close-to-optimal positively and negatively chirped pulses.

It is instructive to look at the dependence of transient absorption on pump pulse energy measured with transform-limited pulses as well as with close-to-optimal negatively and positively chirped pulses (Fig. 6). While the plots for $0 \mathrm{fs}^{2}$ and $-1600 \mathrm{fs}^{2}$ are clearly nonlinear indicating a saturated absorption regime, data for $1200 \mathrm{fs}^{2}$ can be satisfactorily fitted with a straight line. This result serves as an additional confirmation that despite high energy density in the illuminated region of the sample, resonant interactions with positively chirped pulses are primarily of non-saturated (single-photon) nature.

In summary, the results presented herewith highlight the significance of coherent quantum processes in porphycene in solution, which occur despite fast decoherence brought about by condensed phase environment at room temperature. Worth noting is the interplay between coherent (wavepackets) and incoherent (populations) dynamics. On one hand, the degree to which the transient absorption signal can be controlled stresses the importance of keeping the pulses in a nonsaturated regime for standard ultrafast spectroscopic investigations, so as not to i.e. decrease the signal to noise ratio with undesired multi-photon interactions. On the other hand, the results open the door to future studies of influence which coherent interactions in porphycene may have on double hydrogen transfer efficiency and kinetics. Other wavelengths and phase shape combinations could be used, enabling effects such as excited state absorption to contribute, with added flexibility of a shift from an effectively two-level system into a three level one.

This work has been supported by the Polish National Science Centre grant 011/01/B/ST2/02053.

\section{References}

[1] J. Waluk, Porphycenes: Spectroscopy, Photophysics, and Tautomerism. In CRC Handbook of Organic Photochemistry and Photobiology, M. Oelgemoeller, A. Griesbeck, F. Ghetti, Eds. (CRC Press: Boca Raton, FL, 2012), pp 809-829.

[2] P. Fita, N. Urbańska, C. Radzewicz, J. Waluk, Chem. Eur. J. 15, 485 (2009).

[3] P. Ciąćka, P. Fita, A. Listkowski, M. Kijak, S. Nonell, D. Kuzuhara, H. Yamada, C. Radzewicz, and J. Waluk, J. Phys. Chem. B 119, 2292 (2015).

[4] P. Nuernberger, G. Vogt, T. Brixner and G. Gerber, Phys. Chem. Chem. Phys. 9, 2470 (2007).

[5] G. Cerullo, C.J. Bardeen, Q. Wang, C.V. Shank, Chem. Phys. Lett. 262 362 (1996).

[6] C.J. Bardeen, Q. Wang, C.V. Shank, Phys. Rev. Lett. 75, 3410 (1995).

[7] P. Tournois, Opt. Comm. 140, 245 (1997).

[8] W. Demtroder, Laser spectroscopy: basic concepts and instrumentation, 3rd ed. (Springer Press, Berlin, Heidelberg, New York, 2003).

[9] A. Starukhin, E. Vogel, J. Waluk, J. Phys. Chem. A 102, 9999 (1998).

[10] P. Fita, C. Radzewicz, J. Waluk, J. Phys. Chem. A, 112, 10753 (2008). 